\title{
Gyroids of Constant Mean Curvature
}

\author{
Karsten Große-Brauckmann
}

\section{CONTENTS}

\section{Introduction}

1. The Minimal Gyroid and Its Known CMC Companions

2. Evolution by Area under a Volume Constraint

3. Criticality and Stability for Area in CMC Families

4. Evolution of the Gyroid Using the Energy $\int\left(\mathrm{H}-\mathrm{H}_{0}\right)^{2}$

5. Experimental Setup for $\int\left(\mathrm{H}-\mathrm{H}_{0}\right)^{2}$

6. Technicalities of the Evolution

7. All CMC Gyroids

Acknowledgements

References

AMS Subject Classification: Primary 53A10; Secondary 49Q05
We use Brakke's Surface Evolver to deform a triply periodic minimal surface, the gyroid, into a continuous family of constant mean curvature surfaces with the same symmetry. We discuss stability and bifurcation problems for these surfaces.

Minimal surfaces can be rigid if their symmetries are imposed. Without symmetries, however, it is not at all obvious which minimal surfaces are isolated and which are deformable. In the triply periodic case Meeks [1990] proved such a result: the Schwarz $P$ and $D$ surfaces are contained in the same 5-parameter family. In the present paper, we consider a different deformation of a minimal surface: we embed it into a continuous family of constant mean curvature (CMC) surfaces. This oneparameter family is maximal and unique when the (orientation-preserving) symmetries of the minimal surface are imposed.

In numerical work Anderson, Davis, Nitsche, and Scriven [Anderson et al. 1990] embedded five triply periodic minimal surfaces into one-parameter families of CMC surfaces with the same symmetry: these are the Schwarz $P$ and $D$, Schoen $I-W P, F-$ $R D$, and Neovius $C(P)$ surfaces, where we use Schoen's notation. Mathematically Lawson's conjugate surface method [Lawson 1970] with its extensions [Karcher 1989; Große-Brauckmann 1993] provides a tool to prove the existence of these and many other families. Both approaches require the minimal surfaces to have sufficiently many reflectional symmetries. There is one prominent embedded triply periodic minimal surface which does not have any reflections: the gyroid. It is associated to the Schwarz $P$ or $D$ surface, and was discovered by the crystallographer A. Schoen [1970]. A mathematical proof of Schoen's claims was only recently 
given [Große-Brauckmann and Wohlgemuth 1996]. In a sense made precise by its skeletal graph (see Section 1A) the gyroid consists of triple junctions, whereas the Schwarz $P$ surface has sixfold and the $D$ surface fourfold junctions. Since twofold junctions cannot generate triply periodic CMC surfaces, the gyroid is special in that it is the only known triply periodic surface with triple junctions. This explains why we found the CMC gyroid family extreme in a quantitative comparison with the $P$ and $D$ family [Große-Brauckmann 1997].

The gyroid has been mentioned in the context of various microstructure phenomena [Dubois Violette and Pansu 1990]; a general reference for such interfaces is [Nitsche 1989, p. 240]. Recent interest in the gyroid has been stimulated by the fact that interfaces with gyroid symmetries were found in diblock copolymers [Hajduk et al. 1994; Förster et al. 1994]; see also [Thomas et al. 1988] for a survey. Presumably two disconnected interfaces form the polymer interface; in fact the observed symmetry group distinguishes single and double interface.

A model considered for the polymer problem are CMC surfaces. An experimentally observed polymer double interface with gyroid symmetries and a volume fraction of $37.5 \%$ raises the mathematical existence question for a CMC gyroid of volume fraction $18.75 \%$. This problem was posed by the polymer scientist E. Thomas to M. Wohlgemuth and the author, and was not covered by any previous existence results on $\mathrm{CMC}$ surfaces. A first piece of affirmative evidence is given in [GroßeBrauckmann and Wohlgemuth 1996]: the minimal gyroid can be deformed to surfaces with small constant mean curvature, and gyroids of large constant mean curvature exist as well. We conjectured in that paper that the large gap in between these surfaces is in fact bridged by a continuous oneparameter family, containing a surface with the observed volume fraction. The present paper confirms this conjecture numerically, using Brakke's Surface Evolver [Brakke 1992; 1994]. We find gyroids with a volume fraction as low as $5.6 \%$, as described in Section 4.
Surfaces of constant mean curvature are critical points for area under a volume constraint. A complete nonspherical surface is never a minimum to this problem, but if a surface is sufficiently symmetric then its fundamental domain may be small enough to be a stable minimum. At the time our experiments were carried out the Evolver could not invoke rotational symmetries (or work in an orbifold setting); now this feature is available. The Evolver has long been able to work modulo translations, that is, in a 3-torus. Fortunately, by a result of Ross [1992], the gyroid divided by its translation is still stable, and hence, by a result from [Grosse-Brauckmann 1996], it is a minimum of area under volume preserving deformations. The same applies to CMC gyroids close by and this way we obtain the family down to $25 \%$ volume fraction (Section 2). There a bifurcation occurs leading to less symmetric surfaces, which still have the same translations. We observed a similar bifurcation of the CMC $P$ family. It would be interesting to conduct further experiments to understand the bifurcation systematically.

To obtain the remaining part of the family we followed Kusner's suggestion to use a discrete version of the energy

$$
E=\int\left(H(x)-H_{0}\right)^{2} d x \geq 0,
$$

defined on arbitrary surfaces with mean curvature $H(x)$. A surface of constant mean curvature $H_{0}$ has vanishing energy and is hence a minimum of $E$, no matter if it is stable or unstable for area. On the other hand a minimum of $E$ is a surface with constant mean curvature $H_{0}$ provided its energy level is 0 . Critical points with nonzero energy levels do also occur and are a complication for our method. They are known e.g. for compact surfaces under the energy with $H_{0}=0$; Hsu, Kusner, and Sullivan [Hsu et al. 1992] studied such Willmore surfaces with the Evolver.

We believe that many other CMC surfaces can be represented using the Evolver, and a more general goal of this paper is to describe how this can be 


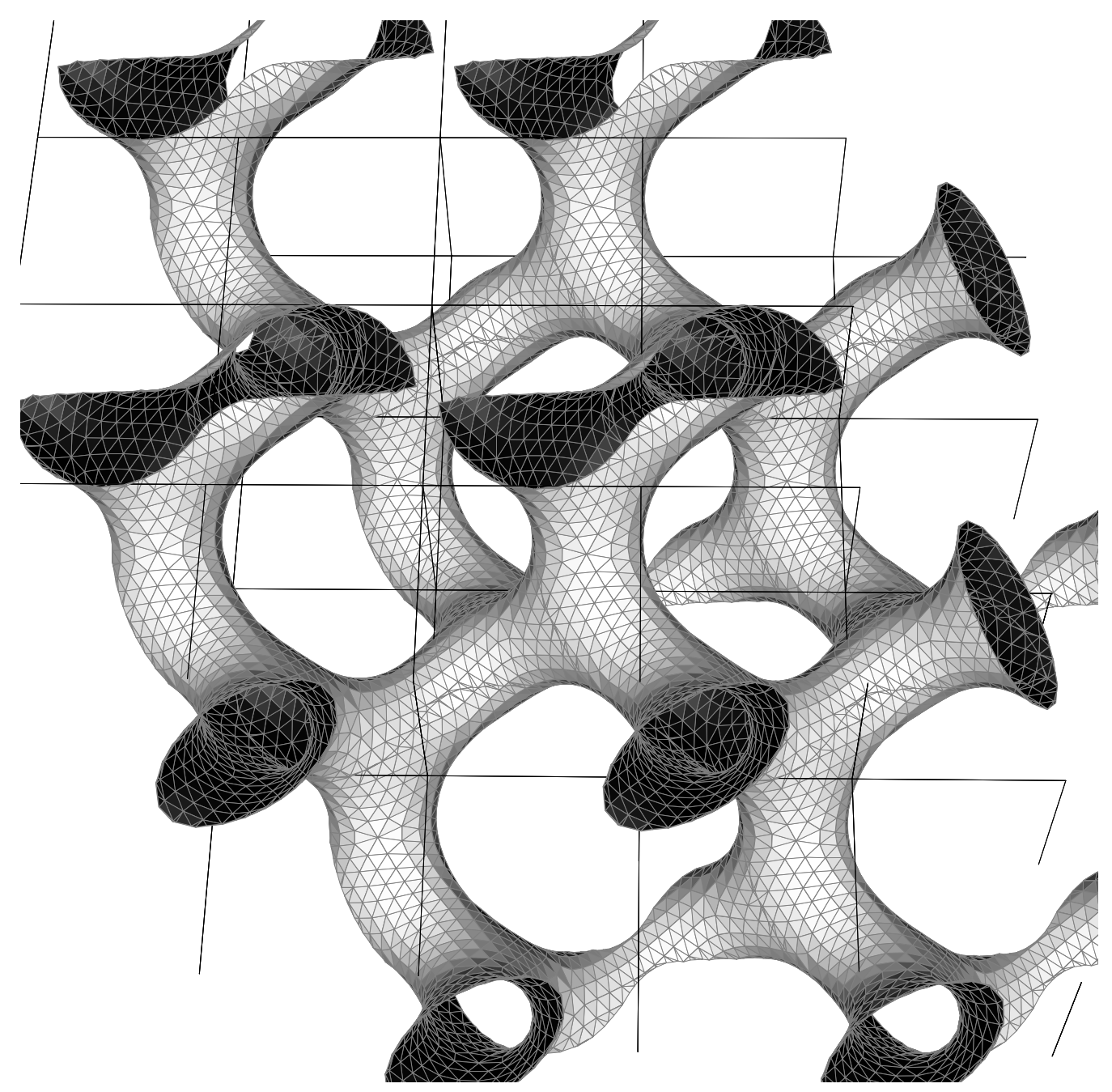

FIGURE 1. A CMC gyroid with volume fraction of $18.75 \%$. At this volume the gyroid is rather tubular and the triple junctions are apparent.

done. A different program specialized to this purpose gives a discrete version of Lawson's conjugate surface construction; it was written by Oberknapp and is included in the package GRAPE [Oberknapp and Polthier 1997]. We used it to study compact and other CMC surfaces in work joint with Polthier [Große-Brauckmann and Polthier 1996; 1997a; 1997b]. For surfaces with sufficiently many reflectional symmetries GRAPE seems to be the more efficient choice, but for surfaces such as the gyroid, or for energies different from surface area, the Evolver can still be used.

\section{THE MINIMAL GYROID AND ITS KNOWN CMC COMPANIONS}

We want to define the symmetry groups and then summarize the results on minimal and CMC gyroids. For details and proofs we refer to [GroßeBrauckmann and Wohlgemuth 1996].

\section{A. Symmetry Groups and Skeletal Graph}

The translational symmetries of minimal and CMC gyroids are given by the body centred cubic (BCC) lattice $\Lambda_{d}$. A symmetric set of generators for this 
lattice consists of the four space diagonals of a cube, $(d, d, d),(d,-d, d),(-d, d, d),(-d,-d, d)$, for fixed $d>0$. A translational fundamental domain is the three-dimensional torus $T_{d}=\mathbb{R}^{3} / \Lambda_{d}$. In our figures we represent the torus by a rectangular box $B_{d}$ of edge lengths $2 d, 2 d, d$, and with identifications $(2 d, 0,0),(0,2 d, 0),(d, d, d)$.

To describe the symmetry groups we first define a set of four points in the torus, which we represent by points in the box $B_{d}$ :

$$
\begin{aligned}
V_{+} & =\left\{\left(\frac{3}{4}, \frac{3}{4}, \frac{1}{4}\right) d,\left(\frac{5}{4}, \frac{1}{4}, \frac{1}{4}\right) d,\left(\frac{3}{4}, \frac{5}{4}, \frac{3}{4}\right) d,\left(\frac{5}{4}, \frac{7}{4}, \frac{3}{4}\right) d\right\} \\
& \subset T_{d} ;
\end{aligned}
$$

we also need

$$
\begin{aligned}
V_{-} & =\left\{\left(\frac{1}{4}, \frac{1}{4}, \frac{3}{4}\right) d,\left(\frac{7}{4}, \frac{3}{4}, \frac{3}{4}\right) d,\left(\frac{1}{4}, \frac{7}{4}, \frac{1}{4}\right) d,\left(\frac{7}{4}, \frac{5}{4}, \frac{1}{4}\right) d\right\} \\
& \subset T_{d} .
\end{aligned}
$$

The box $B_{d}$ decomposes into four little cubes of edge length $d$, and for each cube there is a space diagonal which contains one point of $V_{+}$and one of $V_{-}$, dividing it as 1 to 3 . The umbilics of the minimal and CMC gyroids will be contained in these space diagonals.

Definition 1.1. The CMC gyroid symmetries are the group of isometries of the torus $T_{d}$ leaving $V_{+}$(or $V_{-}$) invariant, and the minimal gyroid symmetries are the group leaving $V_{+} \cup V_{-}$fixed.

These groups do not contain reflections, but they contain rotations of order 2 and 3, and skew rotations of order 4 . The skew rotations result in spiral shapes (Figure 2, right) and, presumably, led to the name gyroid. In crystallographic notation the CMC gyroid symmetries form the group $\mathrm{I}_{1} 32$ while Ia $\overline{3} \mathrm{~d}$ denotes the minimal gyroid symmetries. Each minimal gyroid symmetry which is not a CMC gyroid symmetry can be seen to be orientation-reversing. Such a map has the same effect up to CMC gyroid symmetries; that is it induces a unique involution $\iota$ on CMC gyroid symmetric sets. In particular $\iota$ exchanges the sets $V_{+}$and $V_{-}$.

A skeletal graph can be defined for many symmetric minimal or CMC surfaces and encodes their symmetries and topology [Kusner 1991]. There are two such graphs, one to each side of an embedded surface $M$. They consist of vertices connected by straight lines (edges). Each of the two connected bodies in $\mathbb{R}^{3}-M$ can be retracted to the respective skeletal graph while keeping the symmetry group of $M$. The skeletal graph $S_{+}$of the gyroid is the set of four vertices $V_{+}$plus the six shortest straight lines connecting all pairs of points in $V_{+}$. Likewise the dual skeletal graph $S_{-}=\iota\left(S_{+}\right)$connects the points in $V_{-}$. The lines point in face diagonal directions and have length $d \sqrt{2} / 4$; three lines meet with $120^{\circ}$ angles in each point of $V_{ \pm}$, see Figure 2, left. Definition 1.1 does not change if we replace $V_{ \pm}$by $S_{ \pm}$.

A nice way to generate the CMC gyroid symmetries is to take the set of rotations by $180^{\circ}$ about the edges of the skeletal graph $S_{+}$, which can be seen to agree with the set of the $180^{\circ}$ rotations about the dual skeletal graph $S_{-}$. Such a rotation acts as a transposition on the four points in $V_{+}$(and $V_{-}$). Indeed, it fixes the two endpoints of the edge, and transposes the remaining two points. Thus any permutation of $V_{+}$is generated by a set of three such rotations. Since an isometry of the torus which fixes $V_{+}$is the identity we see that three such rotations generate the group of $\mathrm{CMC}$ symmetries.

\section{B. The Minimal Gyroid}

The minimal gyroid $G$ is associated to the $D$ and $P$ surface, and has hence the same rotational symmetries about the normals of associated points. These axes of rotation are parallel (normals of associated surfaces agree) but their spacing is different. Therefore they generate different symmetry groups. In particular, for $P$ and $D$ the rotations generate reflections, but for the gyroid they do not.

Theorem 1.2. The minimal gyroid is an embedded triply periodic minimal surface with lattice $\Lambda_{d}$ having the minimal gyroid symmetries. It has genus 3 in the torus $T_{d}=\mathbb{R}^{3} / \Lambda_{d}$. The gyroid divides space into two connected components that are related by a reflection and translation represented by $\iota$. The 

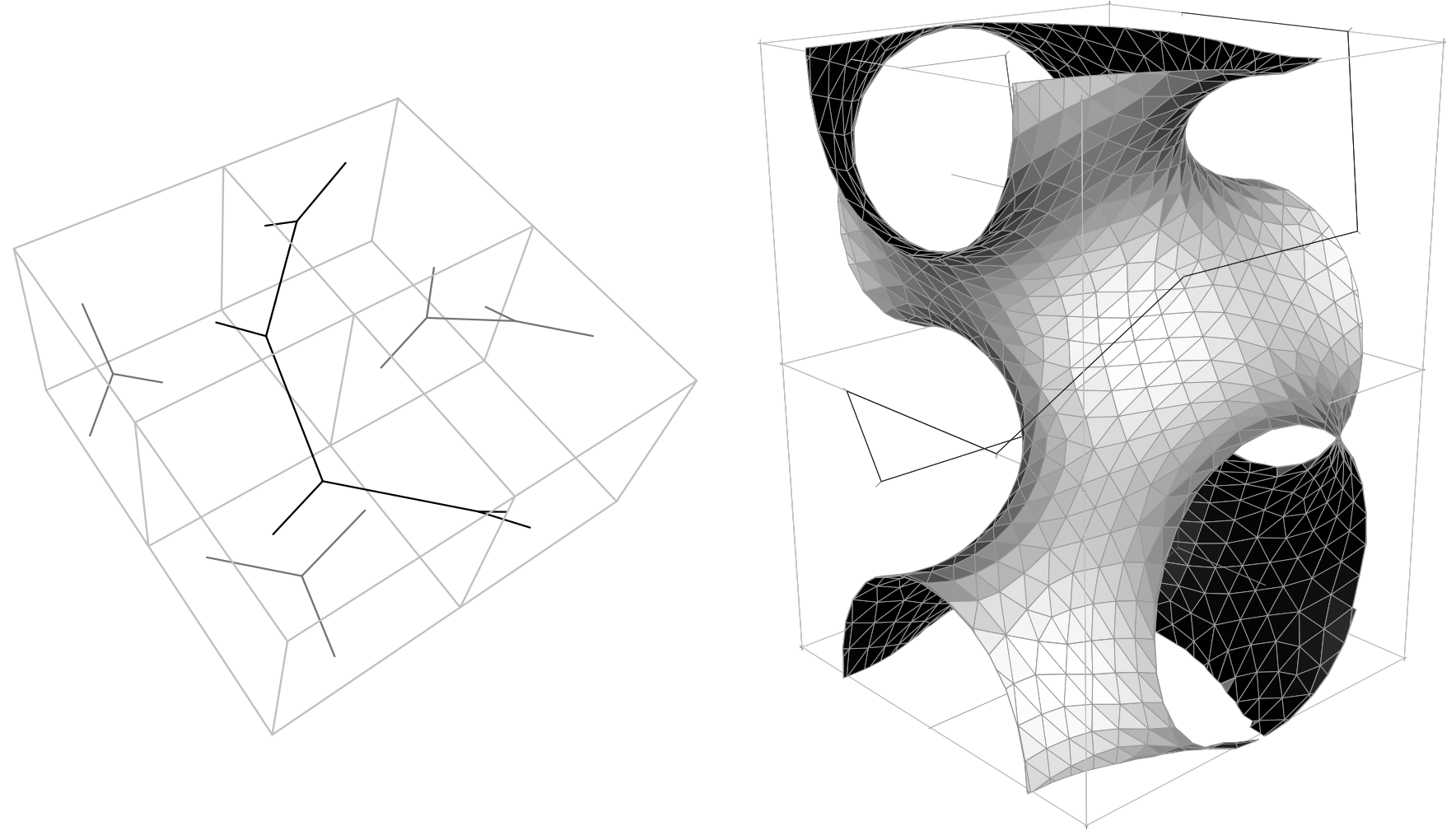

FIGURE 2. Left: The two skeletal graphs $S_{ \pm}$for the gyroid. Each graph has the four nodes $V_{ \pm}$in the body-centred cubic $T_{d}$ outlined by the four little cubes. Each of the six edges of either graph crosses a face of a little cube. Right: A translational fundamental domain of the minimal gyroid. While for the standard $P$-cell there are four horizontal handles at the same height, for the gyroid the horizontal handles gyrate upwards. Indeed, a screw motion of a $90^{\circ}$ rotation about the vertical axis of the rectangular box followed by a vertical shift of a fourth of its height is a symmetry of the surface. Unlike the $P$-cell no face of the box is a plane of reflection. An octagonal helix of one skeletal graph of the gyroid is exhibited outside the surface. Every other edge of the helix is horizontal and contained in a face of the box; together with vertical translations, the $180^{\circ}$ rotations about these edges generate the complete surface. The quadrilateral helix to the interior side of the surface belongs to the dual skeletal graph. The vertical faces of the box in this figure make $45^{\circ}$ angles with those of the figure on the left, while the horizontal faces are parallel.

gyroid $G$ is associated to the Schwarz $P$ and $D$ surface; that is, it can be represented

$$
G=\cos \varphi D+\sin \varphi P
$$

with $\varphi \approx 38.015^{\circ}$; here we add points of $P$ and $D$ that are conjugate to each other, and positioned accordingly in space.

Formula (1.1) can be viewed as the Weierstrass representation formula; it gives the real part of Weierstrass data rotated with $e^{i \varphi}$.
Remark 1.3. In this presentation we considered the minimal gyroid as a set. If instead we view it as an oriented surface (with normal) then its symmetry group is given by the CMC gyroid symmetries. This is a common choice, for instance when the surfaces are represented with the Weierstrass formulas. For $P$ and $D$, but not the gyroid, the choice of symmetry group affects the lattice: the involution $\iota$ for these surfaces can be represented by a translation. The genus- 3 minimal surfaces with such an orientation-reversing translation form Meeks' 5-parameter family mentioned in the introduction. 


\section{C. Constant Mean Curvature Gyroids}

In [Große-Brauckmann and Wohlgemuth 1996] we prove there are gyroids with small mean curvature close to the minimal surface. We also discuss CMC gyroids of large mean curvature, that look like spheres connected with small necks; the existence of these spheroidal gyroids follows from the work of Kapouleas [1990].

Theorem 1.4. There exist $\varepsilon, \delta>0$ such that there is a family of embedded triply periodic surfaces $G_{H}$ with lattice $\Lambda_{d}$ and $\mathrm{CMC}$ gyroid symmetries. For $0 \leq \pm H<\varepsilon$ the family is continuous in $H$, and contains the minimal gyroid $G_{0}$. There exist further gyroids with mean curvature

$$
2 \sqrt{2} / d< \pm H<\delta+2 \sqrt{2} / d .
$$

When $H \rightarrow \pm 2 \sqrt{2} / d$ these spheroidal gyroids converge in Hausdorff distance to a union of four $\mathrm{CMC}$ symmetric spheres of radius $d \sqrt{2} / 4$ in the torus, which we call gyroid close-packed spheres. These spheres have midpoints at $V_{ \pm}$and touch each other in the midpoints of the edges of $S_{ \pm}$.

In the present paper we find a one-parameter family which contains the gyroids of the theorem: the small mean curvature gyroids are in the middle, and the spheroidal gyroids of Kapouleas at the ends. In particular our family also degenerates in gyroid close-packed spheres; at that point, the family cannot be extended any further since handles pinch off and the Gauß curvature blows up. The gyroids of mean curvature $H$ and $-H$ in this family as well as those of Theorem 1.4 are related as follows:

Proposition 1.5. The involution $\iota$ changes the sign of the mean curvature, $\iota\left(G_{H}\right)=G_{-H}$, but leaves the area invariant. The minimal gyroid $G_{0}$ is a fixed point of $\iota$.

We will often refer to the volume of a continuous family $G_{H}$ with gyroid symmetries. By this we mean the volume of one component of $T_{d}-G_{H}$ which is selected in a continuous way. Since $\iota$ interchanges the two components we have

$$
\operatorname{vol}\left(G_{-H}\right)=\operatorname{vol}\left(T_{d}\right)-\operatorname{vol}\left(G_{H}\right)
$$

We call the quotient $\operatorname{vol}\left(G_{H}\right) / \operatorname{vol}\left(T_{d}\right)$ the volume fraction of $G_{H}$. From (1.2) it follows that the volume fraction of the minimal gyroid is a half.

\section{EVOLUTION BY AREA UNDER A VOLUME CONSTRAINT}

A variational characterization of a (compact and boundaryless) CMC surface $M(0)$ in a 3 -manifold is that

$$
\frac{d}{d t}[\operatorname{area}(M(t))]_{t=0}=0
$$

for any smooth family of surfaces $M(t)$ which encloses the same volume as $M(0)$. For the evolution we need minima rather than critical points, and for this reason we consider the second variation. A CMC surface $M$ is called volume preserving stable, or v.p. stable, if for all smooth functions $u$ with $\int_{M} u=0$ on $M$

$$
\int_{M}-u \Delta u-|A|^{2} u^{2} \geq 0
$$

It is called strictly volume preserving stable if the left hand side is larger than $\lambda \int_{M} u^{2}$ for a $\lambda>0$. Here $|A|$ is the norm of the second fundamental form, and $\Delta$ the Laplace-Beltrami operator on $M$. We recall a result of Ross [1992].

Theorem 2.1. A translational fundamental domain of the minimal gyroid, $G / \Lambda_{d}$, is volume preserving stable.

This result can be sharpened to strict stability if the gyroid is fixed with respect to translations; see the proof of Lemma 17 in [Große-Brauckmann and Wohlgemuth 1996]. In [Grosse-Brauckmann 1996] we prove that strict v.p. stability of $M(0)$ implies that the area of $M(0)$ is smaller than that of any 
other close-by surface which encloses the same volume. This makes the minimal gyroid $G \subset T_{d}$ a strict area minimizer among surfaces enclosing the same volume. By continuity of (2.1) we obtain:

Theorem 2.2. The minimal gyroid and sufficiently close deformations to constant mean curvature are strict local minima of area for their respective volume fraction.

Note that the volume constraint is also necessary for a minimal surface: only a planar surface is a minimum of area, but any other minimal surface has larger area than parallel surfaces close by.

Thus we can find CMC gyroids with the Evolver by minimizing area under a volume constraint in the torus $T_{d}$. We found them down to a volume fraction of $25 \%$. In Table 1 we give the data obtained for a triangulation with 1052 vertices, 3168 edges, and 2112 faces. In this and all following tables we choose $d=4^{-1 / 3}$ such that the torus $T_{d}$ has volume 1 . To control the evolution we calculated eigenvalues using the ritz command of the Evolver's hessian method. Clearly the gyroid can be translated in the torus, and thus there is a threedimensional eigenspace with eigenvalue zero. $\mathrm{Nu}$ merically, this eigenvalue had modulus always less than 0.3. Thus the lowest nonzero eigenvalue is the fourth one which has multiplicity 2 . The sixth eigenvalue, although at the minimal surface much larger than the fourth and fifth eigenvalue, comes also close to 0 when these tend to 0 . This could indicate that a bifurcation to further asymmetric solutions occurs close by.

Reducing the volume further resulted in gyroids with fewer symmetries: necks of different sizes appear. Eventually they pinched off. Likewise we observed that the CMC $P$-surfaces bifurcate to less symmetry at a volume fraction of $34 \%$.

Remarks 2.3. 1. Karcher pointed out that these bifurcations could be related to the existence of a known less symmetric $P$-surface with cubic lattice: the stable square catenoids on $P$ [Große-Brauckmann and Wohlgemuth 1996] have a boundary that

\begin{tabular}{|c|c|c|c|}
\hline area & volume & $\begin{array}{c}\text { mean } \\
\text { curvature }\end{array}$ & $\begin{array}{c}\text { least nonzero } \\
\text { eigenvalue }\end{array}$ \\
\hline 2.459 & 0.5 & 0.00 & 8.07 \\
2.442 & 0.437 & 0.27 & 7.62 \\
2.391 & 0.375 & 0.56 & 6.38 \\
2.300 & 0.312 & 0.89 & 4.10 \\
2.239 & 0.281 & 1.08 & 2.61 \\
2.172 & 0.253 & 1.26 & -0.04 \\
\hline
\end{tabular}

TABLE 1. Evolution of the gyroid by area under a volume constraint in a torus of volume 1 .

also bounds an unstable square catenoid. Out of the three handles of $P$, two are still the same under the symmetries of this surface; we do not know if a $P$ surface exists in a cubic cell with all three handles different. In any case we believe that such less symmetric minimal $P$-surfaces can also be embedded into a CMC family; it is unclear if this family bifurcates away from the symmetric family. We can imagine similar desymmetrizations for the gyroid handles.

2. Ross's result gives an analytic reason why the translational symmetries automatically enforce the full symmetry group. We also have a geometric explanation. We expect that a change in diameter of a handle results in a change of its length. This principle is true for the Delaunay surfaces [GroßeBrauckmann and Polthier 1997a] and seems to be generically true for other CMC surfaces. Thus if not all handles are changed the same way then the lattice must change.

\section{CRITICALITY AND STABILITY FOR AREA IN CMC FAMILIES}

Regarding area and volume of a compact CMC family the following simple fact is important.

Lemma 3.1. Suppose $M(t)$ is a smooth family of surfaces such that $M(0)$ has constant mean curvature $H \neq 0$. Then the area of $M(0)$ is critical if and only if the volume is critical. 
Proof. The first variation formula for CMC surfaces states that

$$
\frac{d}{d t}[\operatorname{area}(M(t))-2 H \operatorname{vol}(M(t))]_{t=0}=0 .
$$

We want to apply this lemma to the special case that $M(t)$ is the family of CMC gyroids.

Proposition 3.2. Suppose that the small mean curvature gyroids and the spheroidal gyroids are contained in one smooth family with nonzero mean curvature except at the minimal surface. Then area and volume must be critical for at least one surface and the mean curvature is also critical for at least one surface.

Proof. It follows from Kapouleas' work that we can consider the spheroidal gyroids in highest order as spheres of radius $\sqrt{2} d / 4-r$ (for sufficiently small $r>0)$ joined with necks. These necks have both radius and length of order $O(r)$ so that their area is of order $O\left(r^{2}\right)$, and the volume is of order $O\left(r^{3}\right)$. But both area and volume of the spheres decrease to first order in $r$. This means that in highest order the behaviour of area, volume, and mean curvature is given by the respective quantities for the family of spheres. In particular the CMC family contains surfaces with area and volume fraction less than the close-packed spheres, but larger mean curvature.

The close-packed spheres have a volume fraction of $4(\sqrt{2} / 4)^{3} \approx 0.185$ in the torus which is less than the volume fraction 0.5 of the minimal gyroid $G_{0} \subset$ $T_{d}$. Thus if these two subfamilies are bridged by a smooth one-parameter family there must be a surface with critical volume in between, which, by the previous lemma, has critical area or vanishing mean curvature. A similar continuity argument applies to the mean curvature.

We believe, but do not know, that the minimal gyroid is unique in the class of CMC surfaces of genus 3 with CMC gyroid symmetries. In that case the nonvanishing assumption on the mean curvature in the proposition could be replaced by a symmetry assumption.

We want to relate the existence of a surface with critical area and volume to stability properties of the family. Weaker than stability in the torus is to require stability on a smaller domain:

Definition 3.3. We call a CMC surface $r$-stable if it is v.p. stable with respect to variations respecting the (full) symmetry group of the surface.

When $\mathbb{R}^{3}$ is divided by such a symmetry group, in general an orbifold results, and $r$-stability is the stability of the surface in this orbifold. Note that for the gyroid the orbifold does not allow translations any more, and thus a gyroid can be strictly $r$-stable. Since the Evolver does not (yet) allow us to invoke rotational symmetries we could not test on $r$-stability numerically. In any case we have the following mathematical facts.

Theorem 3.4. (i) The CMC gyroids are strictly $r$ stable in a neighbourhood of the minimal gyroid.

(ii) Close to the gyroid close-packed spheres the spheroidal $\mathrm{CMC}$ gyroids are r-unstable.

(iii) A CMC gyroid which is critical for both area and volume (in the $\mathrm{CMC}$ symmetric class) is not strictly $r$-stable.

A similar theorem holds for the $P$ and $D$ surface. Presumably, it applies to many more CMC surface families obtained from minimal surfaces.

Proof. The first statement is a special case of Ross's Theorem 2.1.

To prove the second statement we consider a blow up of the spheroidal gyroids as follows. Each handle has a shortest closed geodesic. We rescale the surfaces such that the length of this geodesic is one. If we position the CMC gyroids such that the geodesics are always contained in a unit ball, then by the work of Kapouleas a subsequence smoothly converges to a catenoid with waist circumference one.

As we shall see in Lemma 3.5 below, the catenoid is not stable for area under a volume constraint. 
The instability arises from a rotationally symmetric eigenfunction with a negative eigenvalue. This eigenfunction respects all the CMC gyroid symmetries. Thus the gyroid neck with symmetries is asymptotically unstable. By the smooth convergence, CMC gyroids close to the gyroid sphere limit are unstable.

The third statement follows from the fact that the one-parameter family at this surface gives rise to a nontrivial variation which preserves the volume. Hence the surface cannot be strictly stable.

Lemma 3.5. The catenoid is not volume preserving stable among (compactly supported) variations which respect all its symmetries.

Proof. This follows from the instability of the Delaunay surfaces proved by Athanassenas [1987]. We sketch a more explicit proof. The catenoid is a surface of revolution, obtained by rotating a graph $r(z)$ about the $z$-axis. A variation $r(t, z)$ with $r(0, z)=r(z)$ which is symmetric in $\pm z$ obviously respects the catenoid symmetries. It is well-known that the catenoid is unstable for area alone, and thus there is a (symmetric) variation that decreases area in first order. This variation changes the enclosed volume, but we can compensate for it with an arbitrary small change of area when we move out far enough to infinity. To see this, note that the area of a surface of revolution is $2 \pi \int r \sqrt{1+r^{\prime 2}}$ whereas the volume is $\pi \int r^{2}$ (integration over a compact $z$-interval). This variation can be taken with compact support disjoint to the area decreasing variation.

It follows from the lemma that none of Kapouleas' surfaces is stable for its symmetry group.

\section{EVOLUTION OF THE GYROID USING THE ENERGY $\int\left(\mathrm{H}-\mathrm{H}_{0}\right)^{2}$}

We obtained a maximal continuous family of constant mean curvature gyroids using the energy

$$
\int\left(H-H_{0}\right)^{2}
$$

where we prescribed $H_{0}$ suitably. In this section we describe the geometry of the CMC gyroid family, in the next our Evolver settings, and thereafter we give a more technical description of the evolution.

Our main result is that the simplest situation allowed by Proposition 3.2 occurs: up to $\iota$ there is only one surface critical for area and volume, and also only one with critical mean curvature. A similar statement holds for the five surfaces studied by Anderson [1990].

Experimental Result 4.1. There is a maximal continuous one-parameter family $\mathcal{G}=\iota(\mathcal{G})$ of embedded triply periodic surfaces, called CMC gyroids, with lattice $\Lambda_{d}$ and the CMC gyroid symmetry group. The family includes the gyroids of Theorem 1.4. It contains two $\iota$-related least-area surfaces which also have extremal volume, and two $\iota$-related surfaces with maximal $|H|$. The family is not closed, and has two degenerate endpoints given by the two $\iota$-related gyroid close-packed spheres.

In Table 2 we give data obtained for a triangulation with 764 vertices, 2304 edges, and 1536 faces; for the last three rows we had to refine this triangulation. We add in italics values for two exact surfaces: these are the minimal surface (using that the $P$ surface contained in a unit cube has area 2.3451 [Anderson et al. 1990, p. 361]) and the gyroid close-packed spheres.

Like Anderson [1990] we did not study the family in between the maximal mean curvature surface and the gyroid close-packed spheres too well; see Section 6 F. In Figure 3 we give plots of our data in a format similar to the plots in [Anderson et al. 1990]. In the plots we extended the half-family we computed to the entire family by the involution $\iota$ of Proposition 1.5. Note that the slope of the area to volume graph is exactly twice the mean curvature. Indeed, on closed intervals we can parameterize the family by its volume as $\operatorname{vol}(M(t))=t+$ const. If $H(t)$ is the mean curvature of $M(t)$ then the first variation formula (3.1) implies the claim

$$
\frac{d}{d s}[\operatorname{area}(M(s))]_{t}=2 H(t) .
$$




\begin{tabular}{|ll|l|l|l|}
\hline area & $\begin{array}{c}\text { relative } \\
\text { area }\end{array}$ & volume & $\begin{array}{c}\text { mean } \\
\text { curvature }\end{array}$ & \\
\hline 2.4537 & 1 & 0.5 & 0 & exact minimal gyroid \\
2.461 & 1.002 & 0.497 & 0 & see Figure 2 and Figure 4, top left \\
2.378 & 0.969 & 0.362 & 0.63 & \\
2.186 & 0.891 & 0.256 & 1.27 & \\
1.979 & 0.807 & 0.188 & 1.84 & see Figure 1 and Figure 4, top right \\
1.742 & 0.710 & 0.134 & 2.54 & \\
1.415 & 0.577 & 0.080 & 3.81 & \\
1.298 & 0.529 & 0.066 & 4.44 & \\
1.200 & 0.489 & 0.056 & 5.29 & least area and volume: see Figure 4, bottom left \\
1.350 & 0.550 & 0.069 & 5.52 & maximal mean curvature \\
1.453 & 0.592 & 0.079 & 5.40 & see Figure 4, bottom right \\
2.494 & 1.016 & 0.185 & 4.48 & gyroid close-packed spheres \\
\hline
\end{tabular}

TABLE 2. The one-parameter family of CMC gyroids in a torus of volume 1.

Geometrically it seems that the minimal gyroid shrinks to CMC gyroids which look much like tubes about the skeletal graph $S_{+}$around a volume fraction of a fifth. After that, necks shrink into the tube at the middle of the edges of $S_{+}$. When the minimum in area and volume is reached the surfaces look like a union of thickened equilateral tri- angles (lying in the tangent plane of $S_{+}$at $V_{+}$). These triangles thicken further to spheres on the way to the gyroid close-packed spheres while the connecting necks shrink. In Figure 4 we display CMC gyroids for four different volume fractions.

We return to the stability discussion of the previous section. Theorem 3.4 implies that the arc
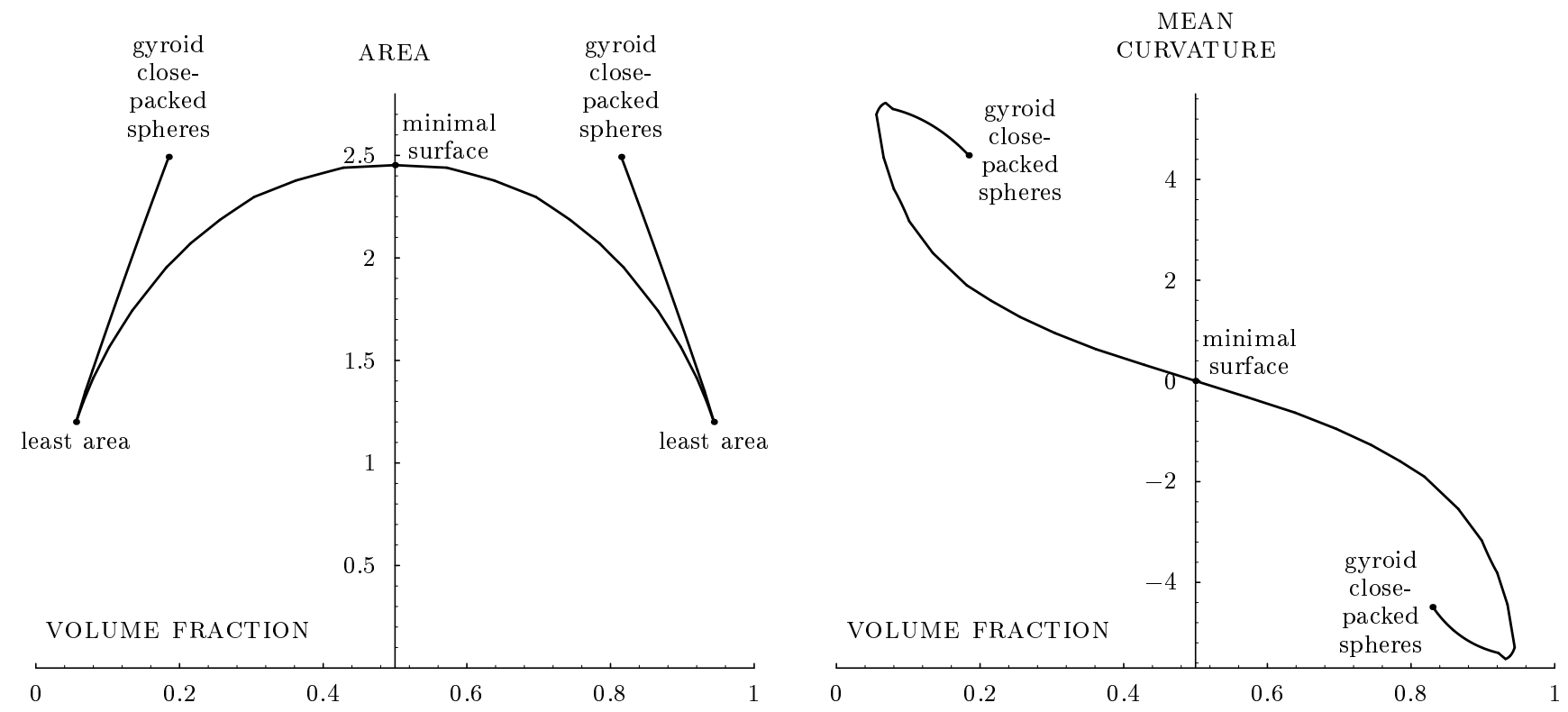

FIGURE 3. Area, volume, and mean curvature of the CMC gyroid family, in a torus of volume 1 . The mean curvature is normalized to equal 1 for the unit sphere. Bumpy spots reflect insufficient data; see Section $6 \mathrm{~F}$. 

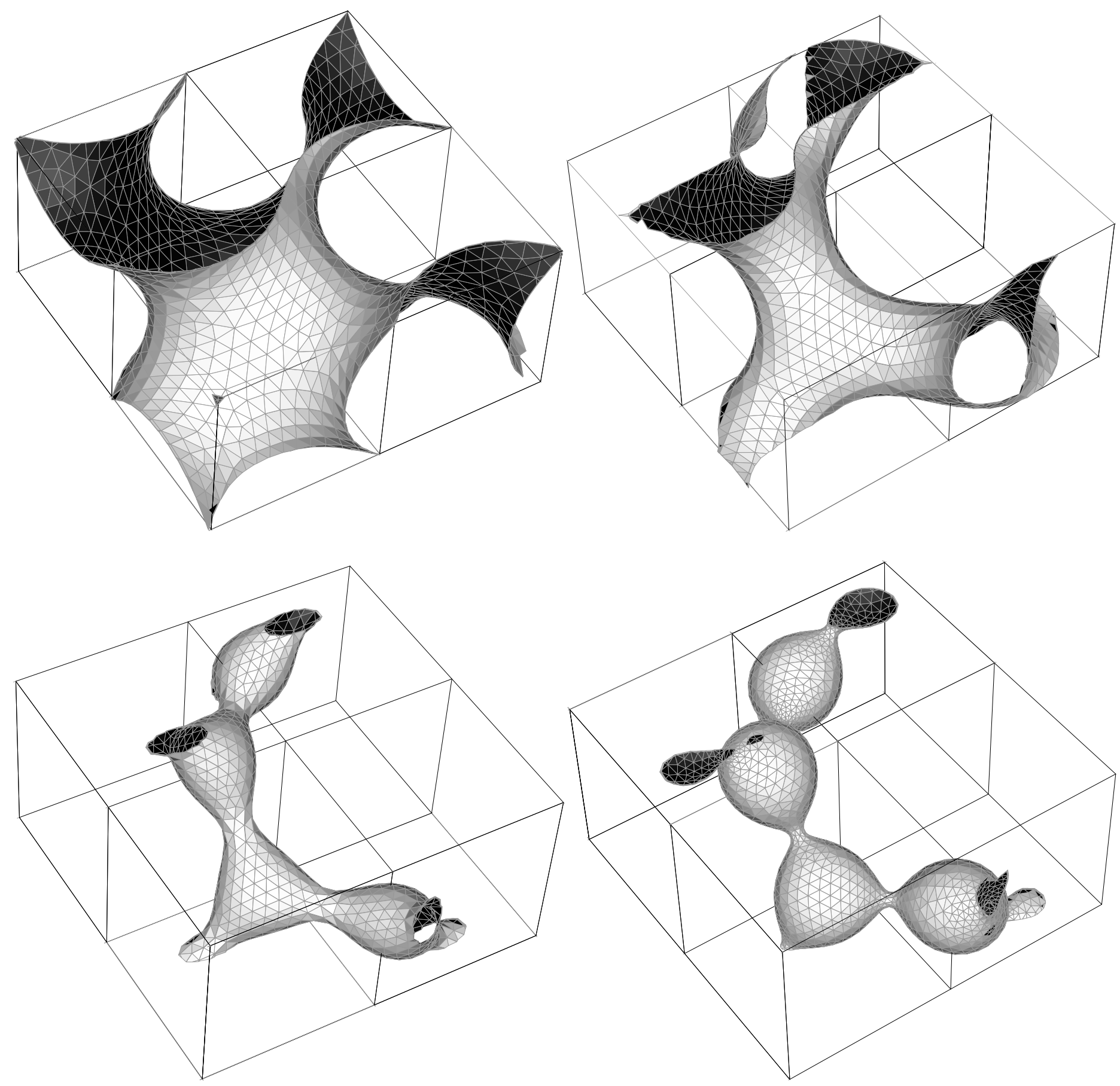

FIGURE 4. Fundamental domains for gyroids with different volume fractions. Top left: The minimal gyroid (volume fraction 50\%). Top right: A CMC gyroid with volume fraction 18.75\%, which looks much like tubes about the skeletal graph. Bottom left: The least-area CMC gyroid, which also has the smallest volume fraction $5.6 \%$ in our CMC family. Bottom right: A spheroidal CMC gyroid with volume fraction $7.9 \%$. 
of the CMC gyroids containing the minimal gyroid can at most be $r$-stable up to the least-area surface. We believe that $r$-stability is lost exactly at this surface.

Conjecture 4.2. The CMC gyroid family is strictly $r$ stable for area under a volume constraint from the minimal surface up to the least-area surface.

A similar conjecture can be made for all surfaces to which Theorem 3.4 applies, in particular $P$ and $D$. For those two surfaces further evidence to the conjecture is given by the fact that no bifurcation to surfaces with the same symmetry can occur in between the minimal and the extremal surface; this result follows with the methods of [Große-Brauckmann 1993]. This evidence is not conclusive, however, since a possible zero eigenvalue in between the minimal and the least-area surface could correspond to a nonintegrable Jacobi field.

Remark 4.3. Physically it is interesting to study families of minimizers with gyroid symmetries for other energies, such as the squared mean curvature integral $W(M)=\int_{M} H^{2}(x)$ under a volume constraint; compare [Hsu et al. 1992]. Among the Delaunay surfaces the cylinder is likely to be favoured by $W$ (for given volume per period, say). The tube-like surfaces in our CMC gyroid family are the best analogue to the cylinder since apparently their Gauss curvature is distributed in the most uniform way among the family. Thus it is reasonable to expect these surfaces to be close to minimizers of $W$. Indeed, evolution under $W$ of the CMC gyroid with $18.75 \%$ volume fraction resulted in an almost unchanged surface with area 1.975 and energy 6.62 (in a triangulation with 6624 faces). Further experiments would be interesting and could lead to a more precise conjecture for the Delaunay surfaces.

\section{EXPERIMENTAL SETUP FOR $\int\left(\mathrm{H}-\mathrm{H}_{0}\right)^{2}$}

The evolution with $\int\left(H-H_{0}\right)^{2}$ gives CMC surfaces which need not be stable for area under a volume constraint. We have to pay for this gain with slower convergence and problems with degeneracies. Thus the energy $\int\left(H-H_{0}\right)^{2}$ should be only used when the evolution by area under a volume constraint fails. Note that the area is computed by integrating first derivatives, whereas for the mean curvature $H$ second order derivatives must be calculated.

We hope that the following remarks together with the Evolver manual [Brakke 1994] make it possible to reproduce our results.

We started the Evolver in its "quantities" version with evolver -q. The discrete form of the energy $\int\left(H-H_{0}\right)^{2}$ is calculated in the Evolver as follows. A vertex $v$ has a star of triangles around it with area $A_{v}$. The gradient with respect to the position of the vertex in space gives a force $F_{v}=$ $-\nabla A_{v}$. The gradient $N_{v}$ of the volume spanned by the star at $v$ is a quantity similar to area times the normal at the vertex. For reasons explained in [Brakke 1994, §7.3.9] a numerically well-behaved definition of the mean curvature at $v$ is

$$
H_{v}=\frac{3}{2} \frac{\left|F_{v}\right|^{2}}{N_{v} \cdot F_{v}},
$$

where $N_{v}$ is the volume gradient of the star. This mean curvature is effected with normal curvature on. Thus the discrete energy is the sum of the vertex energies weighted with their area contribution,

$$
E=\sum_{\text {vertices } v}\left(\frac{3}{2} \frac{\left|F_{v}\right|^{2}}{N_{v} \cdot F_{v}}-H_{0}\right)^{2} \frac{A_{v}}{3} .
$$

It is active with

quantity star_nh2 energy global_method star_normal_sq_mean_curvature

in the input file header. The offset $H_{0}$ in mean curvature, which is our free parameter for the CMC gyroid family, is specified by the parameter $\mathrm{h} z$ zero declared in the input file, and changed interactively using the Evolver's A command.

In order to have the area displayed, we set

$$
\text { default_area_modulus := } 1
$$

but avoided having it contribute to the energy functional with set facet tension 0 . We also desired the Evolver to display the volume using the 
v command. Since the volume calculation of the Evolver via the divergence theorem is ambiguous in a 3-torus by integer multiples of a sixth of the torus volume it is necessary to state the actual volume in the input data file up to a sufficiently small error. The volume is included in the data file after the body; in order to have the Evolver not consider this a constraint, unset body target (or the $\mathrm{b}$ command with parameter 0 ) must be declared. Note that by the same token an iteration step should never change the volume by more than a sixth of the torus volume.

Brakke wrote a hessian routine for the star_nh2 energy in the course of our experiments. At present it can only be used for surfaces without boundary. We invoked the toggle hessian_normal on to restrict the hessian to normal motions. The hessian works for sufficiently small energies (order $o\left(10^{-7}\right)$ ) reliably, and quickly reduces the energy further. Having check_increase on it is worth trying the hessian for larger energy levels too, as it moves the surface in one step as much as perhaps only thousands of standard gradient steps do. This results very often in a considerable geometrical change of the surface, even if the energy does not drop much. Note that in a gradient step information does not spread further than one triangle, whereas the second order methods solve the equation all at once. The same applies to the saddle command which we also used occasionally. Problems of the hessian with large data files were avoided by ysmp off.

Evolution by the star_nh2 energy is rather sensitive to the quality of the triangulation. An even triangulation is easily produced by the Evolver's $\mathrm{w}, \mathrm{u}, \mathrm{l}$, t commands, followed by a smoothing $\mathrm{V}$. This worked well from the minimal to the leastarea CMC gyroid. For the surfaces with small necks, however, the triangulation needs to be refined at regions of high Gauss curvature (compare Figure 4, lower right). Too coarse triangulations seem to deteriorate. To refine the necks we carefully iterated the commands $\mathrm{n}, \mathrm{u}, \mathrm{K}$, followed by some V's; if necessary we invoked the $t$ command to make sure the edge lengths would not vary by more than a factor of 10 .

\section{TECHNICALITIES OF THE EVOLUTION}

\section{A. Initial Surfaces and Gyroid Foam}

The vertices $V_{+}$of one skeletal graph form a subset of the face centred cubic (FCC) lattice which has order 4 in the body-centred cubic (BCC) lattice $\Lambda_{d}$ of the gyroid. To see this, it is convenient to translate $V_{+}$by $(d / 4, d / 4, d / 4)$. All translated vertices sit on the midpoints of the edges and in the centres of the smaller cubes; that is, they form a subset of an FCC lattice with respect to the smaller cubes. A consequence is that the Voronoi cells of the FCC lattice, rhombic dodecahedra, can be used as an initial surface for evolution: rhombic dodecahedra placed at the vertices of one skeletal graph touch three other rhombic dodecahedra, and popping the faces in common results in a polyhedral surface with CMC gyroid symmetries.

A more convenient way to obtain an initial surface is Voronoi Cell Solver (VCS) written by J. Sullivan [1992]. The eight Voronoi cells of $V_{+} \cup V_{-}$are 17-faced polyhedra, with 2 hexagons, 12 quadrilaterals, and 3 decagons; see [Schoen 1970, Fig. II-2a]. With the vCS program faces can be popped. When we pop the twelve decagons an initial surface with minimal gyroid symmetries results. We would like to mention that the relaxed Voronoi cells form a gyroid foam that is far from optimal in regard to cell area: the gyroid cell (normalized to unit volume) has area 5.665, whereas Kelvin has 5.306 and Weaire-Phelan 5.288 (data courtesy of J. Sullivan, personal communication).

\section{B. Bifurcation Points}

In Section 2 we mentioned the existence of a bifurcation at about a volume fraction of $25 \%$. For an evolution using $\int\left(H-H_{0}\right)^{2}$ this bifurcation also appeared and led to the undesired arc of surfaces with less rotational symmetry. Although the Evolver does not allow us to enforce the full symmetry group directly, the following achieves the 


\begin{tabular}{|c|c|c|}
\hline area & volume & $\begin{array}{c}\text { mean } \\
\text { curvature }\end{array}$ \\
\hline 1.199589 & 0.056435 & 5.283 \\
1.199568 & 0.056433 & 5.289 \\
1.199566 & 0.056432 & 5.292 \\
1.199570 & 0.056433 & 5.296 \\
\hline
\end{tabular}

TABLE 3. The third row gives data for the least-area gyroid, and the remaining rows for gyroids nearby.

same effect. With a coarse triangulation we adjusted $H_{0}$ step by step to pass the bifurcation point. Since our initial surface has the CMC gyroid symmetries its evolution is also symmetric. Hence only added numerical inaccuracies lead from a symmetric initial surface to an asymmetric evolution. Once past the bifurcation point, refinement did not cause any problems. In particular we did not encounter any further bifurcations.

We remark that with a too coarse evolution the discrete surfaces evolve into a family with monotone area and volume, so that there is no spheroidal arc. Thus some refinement is necessary to produce the qualitative behaviour of the smooth case.

\section{C. The Least-Area Gyroid}

The Evolver's hessian provides a fast method to trace a minimizer upon variations of a functional. Here we are interested in determining minimizers of the energy $E=E_{H_{0}}$ upon variations of the constant $H_{0}$. Suppose that a surface $M\left(H_{0}\right)$ minimizes $E_{H_{0}}$ for some value $H_{0}$, in the sense that $E_{H_{0}} \ll 10^{-7}$. Then there is an $\varepsilon>0$ such that the same surface still has energy $E_{H_{0}+\varepsilon}<10^{-7}$. Thus the hessian can be invoked for the energy $E_{H_{0}+\varepsilon}$. It will quickly reduce energy further and produce a new minimizer $M\left(H_{0}+\varepsilon\right)$. This procedure can be iterated as long as the triangulation does not deteriorate. Even though one needs to work with small steps in $H_{0}$, this is much more efficient than to work with standard gradient steps.

We used this method to determine the leastarea gyroid, which is illustrated in Figure 4, lower left. Table 3 indicates that as in the smooth case (Proposition 3.2) the same polyhedral CMC gyroid attains least area and extremal volume provided the combinatorial type is left unchanged.

\section{D. Size of Error}

The hessian steps described in the last subsection allow one to determine the discrete $\mathrm{CMC}$ gyroid with least area precisely. However, the result depends on the triangulation chosen. To judge how significant our data are for the smooth case we need to estimate the error arising from discretisation. As an example we studied the effect of refinement for the least-area gyroid, Table 4 gives the result.

The first row of the table represents a triangulation of the fineness of Table 2, the last row the fineness used for Table 3. Another test for our results is the minimal surface: Area and volume of our evolved minimal surface with prescribed mean curvature 0 deviate from the "exact" figures by $0.3 \%$ (see Table 2).

Although we cannot give precise error estimates, we think that the data for area and volume in the Table 2 are no more than 1\% apart from the values for smooth surfaces. Certainly the Evolver can handle a triangulation as fine as Anderson's triangulation of 38000 faces, but to trace an entire one-parameter family becomes time-consuming.

In Table 4 we show two figures that we consider significant for the quality of a triangulation: the

\begin{tabular}{|c|c|c|c|c|c|c|}
\hline area & volume & \#vertices & \#edges & \#faces & $\begin{array}{l}\text { dihedral } \\
\text { angle }\end{array}$ & $\begin{array}{l}\text { length } \\
\text { quotient }\end{array}$ \\
\hline 1.207 & 0.05642 & 764 & 2304 & 1536 & 43 & 1.7 \\
\hline 1.203 & 0.05664 & 2530 & 7602 & 5068 & 23 & 2.3 \\
\hline 1.200 & 0.05643 & 4955 & 14919 & 9946 & 15 & 2.1 \\
\hline
\end{tabular}

TABLE 4. The effect of refinement for a fixed mean curvature $H=5.289$. 
maximal dihedral angle between two adjacent triangles, and the maximum quotient of edge lengths occurring in a triangle. The latter figure indicates how much the worst triangle in the triangulation deviates from being equilateral.

\section{E. Maximal Mean Curvature}

Since we used the mean curvature as a parameter the determination of the maximum in mean curvature needs to be explained. On increasing $H_{0}$ we eventually reached a value for which the energy did not approach 0 any more. We interpreted this to be the maximal mean curvature in the family. To follow the family further we prescribed a slightly larger volume with $\mathrm{b}$ for a few iteration steps. Then we resumed the standard mode with a somewhat smaller prescribed mean curvature. The result were surfaces with larger area and volume than before, so that we would follow the family on the arc towards the spheroidal gyroids. This determination of the maximum of mean curvature in the family seems to be more dependent on the triangulation (and hence less precise) than the determination of least area and volume.

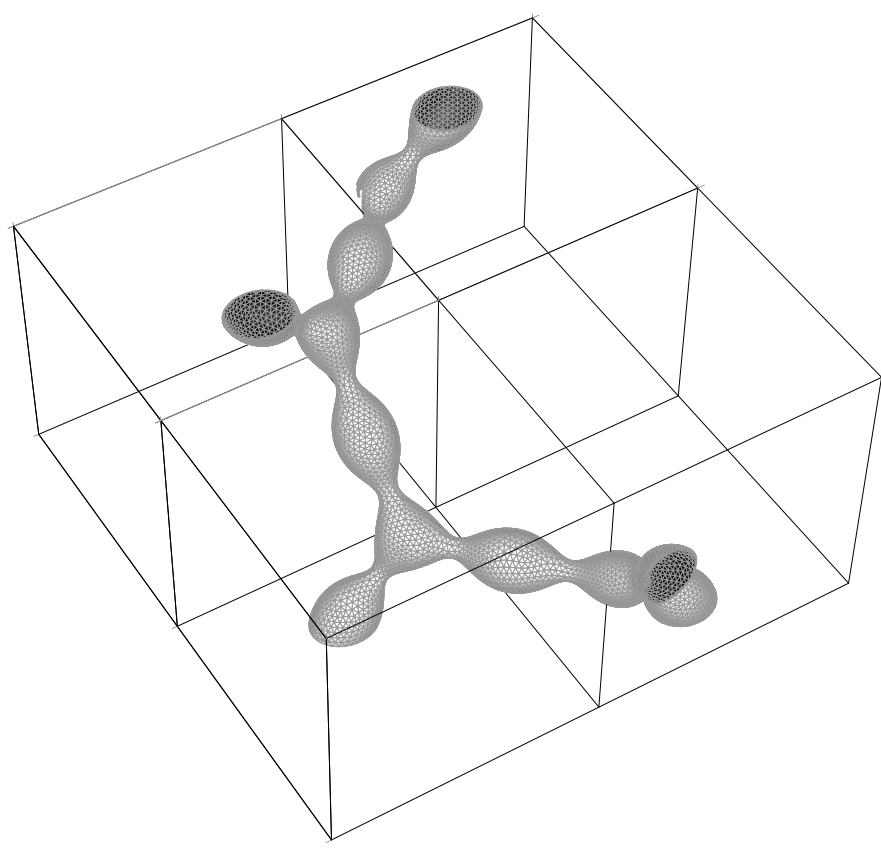

FIGURE 5. A CMC gyroid with one extra bubble.

\section{F. The Spheroidal Gyroids}

Following the family to the gyroid close-packed spheres is complicated for two reasons: small necks require very small triangles and cause numerical problems, and the energy does not vary much between surfaces of very different areas and volumes. This made convergence rather slow. Also, we could not prescribe large changes in $H_{0}$ as it seemed the evolution did not find a close-by CMC surface any more. This problem appeared in surfaces with nonzero stationary energy levels. We believe it is possible to follow the spheroidal gyroids further; since the sphere limit is well-understood theoretically we accepted the gap between our last surface in Table 2 and the gyroid close-packed spheres.

\section{ALL CMC GYROIDS}

As Figure 5 shows there are more CMC gyroids than just those described in our Experimental Result 4.1. The depicted surface has area 0.69 , a volume fraction of only $1.56 \%$, and mean curvature 12.7 (in a triangulation with 11528 faces). This surface could be called a gyroid with one extra bubble (on the edges of the skeletal graph); we want describe in this section that we similarly expect CMC gyroids with any number $n \in \mathbb{N}$ of bubbles on the edges.

By a CMC gyroid we mean a triply periodic surface with lattice $\Lambda_{d}$, genus 3 in the torus $\mathbb{R}^{3} / \Lambda_{d}$, and invariant under the CMC gyroid symmetries. We also assume embeddedness in the slightly generalized form of almost embeddedness (see [GroßeBrauckmann and Kusner 1996] for a definition). All such CMC gyroids obtained with the method of [Kapouleas 1990] can be described as follows.

Theorem 7.1. For each integer $n \in \mathbb{N}_{0}$ there exist $\mathrm{CMC}$ gyroids with mean curvature in the interval $\sqrt{2}(2 n+2) / d< \pm H<\sqrt{2}(2 n+2) / d+\varepsilon$ for some $\varepsilon(n)>0$. The degenerate limit for $H \rightarrow$ $\pm \sqrt{2}(2 n+2) / d$ are Kapouleas spheres: a union of $6 n+4$ spheres of radius $\frac{1}{2} \sqrt{2} d /(2 n+2)$ such that there are $n+2$ centres of spheres equally spaced on 
each edge of the skeletal graph $S_{ \pm}$, with the outermost centres contained in $V_{ \pm}$.

According to Kapouleas (personal communication) a proof of continuity for these families could be worked out. At the Kapouleas spheres the families stop naturally, but we think they can be extended over the other end up to another degenerate surface, which is again a union of spheres. More precisely, from similar cases [Große-Brauckmann 1993] we expect the following maximal continuous families (which include Kapouleas' surfaces).

Conjecture 7.2. All CMC gyroids are given by a countable union of continuous one-parameter families $\mathcal{G}_{n}^{ \pm}, n \in \mathbb{N}$, as well as the family $\mathcal{G}$ from our Experimental Result 4.1. The families with different superscript \pm differ by the involution $\iota$, so that $\iota\left(\mathcal{G}_{n}^{ \pm}\right)=\mathcal{G}_{n}^{\mp}$. The two degenerate endpoints of each family $\mathcal{G}_{n}^{ \pm}$are given by a union of spheres: one are the $6 n+4$ Kapouleas spheres of the previous theorem, and the other the 6 n noidal spheres of radius $\frac{1}{2} \sqrt{2} d / 2 n$, with $n$ centres on each edge of the skeletal graph $S_{ \pm}$.

For the noidal spheres the outermost spheres on the edges touch exactly in the vertices of $V_{ \pm}$. The term noidal refers to the character of the triple junction: a suitable blow-up at $V_{ \pm}$of the surfaces close to the spheres converges to minimal trinoids (minimal surfaces with three catenoid ends in dihedral symmetry).

Note that if a Kapouleas gyroid with mean curvature $H$ is contained in $\mathcal{G}_{n}^{+}$then its involutive image with mean curvature $-H$ is in a different connected component of the moduli space, namely in $\mathcal{G}_{n}^{-}$. Only the family $\mathcal{G}$ of our Experimental Result 4.1 is invariant under the involution $\iota$; the geometric reason for this difference is that starting with gyroid close-packed spheres (Kapouleas spheres for $n=0$ ), there are no spheres left for it to end in noidal spheres. Instead $\mathcal{G}$ contains the minimal gyroid, and continuous with the $\iota$-symmetric arc to reach the other gyroid close-packed spheres.
How do we expect volume and area to behave on the families?

Conjecture 7.3. Each family $\mathcal{G}_{n}^{ \pm}$contains one surface with least area and least enclosed volume, it also contains one surface with maximal $|H|$. Furthermore, for $n \rightarrow \infty$ each sequence $M_{n} \in \mathcal{G}_{n}^{ \pm}$converges in Hausdorff distance to the skeletal graph $S_{ \pm}$. In particular area $\left(M_{n}\right)$ and $\operatorname{vol}\left(M_{n}\right)$ converge to 0 .

The evidence for this conjecture is a reasoning similar to the proof of Proposition 3.2: area and enclosed volume at the spherical endpoints of the family are larger than that of CMC surfaces close by. A critical point for the area must occur, which by Lemma 3.1 is expected to be critical for the volume too. Similarly for the mean curvature. On the assumption that the minimum in mean curvature of each family $\mathcal{G}_{n}^{ \pm}$converges to infinity with $n \rightarrow \infty$ the statement for the sequence $M_{n}$ follows from the tubular enclosure theorem and the area bounds given in [Korevaar and Kusner 1993].

We would like to conclude our paper with some suggestions for experimental work. It would be interesting to understand the bifurcation mentioned in Section 2. Moreover it can be asked if further bifurcations exist and what they lead to. It might also be possible to get some understanding of the class of all CMC surfaces of genus 3 in $T_{d}$. We expect this class to be rather large since Meeks [1990] constructs a sequence of embedded minimal surfaces with genus 3 in some fixed torus with unbounded area. To make the geometry of Meeks' surfaces more concrete would be another worthwhile project; not to mention their possible $\mathrm{CMC}$ deformations.

Remark 7.4. Currently we study deformations of CMC surfaces with triple junctions [Große-Brauckmann and Kusner 1996; Große-Brauckmann and Polthier 1997b]. The surprising result of that work is that bubbles can be generated or deleted on the edges of the skeletal graph if we allow their angles at the triple junctions to vary. Fixing $H=1$ this 
means that the length of the edges is at our disposition if the lattice (or the torus) is not fixed in such a deformation. Thus it seems that there is great freedom for deformation in the class of triply periodic CMC surfaces; it may be that all CMC surfaces of genus 3 are contained in one connected component of the respective moduli space. To summarize let us distinguish two cases: when symmetries are imposed, there are different connected components of the moduli space, characterized by the number of bubbles on the edges (Conjecture 7.2); however, without any symmetry assumptions there might be just one such component. The in-between case of only translational symmetries seems especially interesting; we expect a discrete set of set of surfaces for prescribed $H$.

\section{ACKNOWLEDGEMENTS}

This paper was written while the author visited GANG at University of Massachusetts, Amherst, during the Summer of 1995. It is a completely revised form of GANG preprint IV.13. We thank Rob Kusner for the invitation to GANG, and Ken Brakke for his advice and improvements made to the Evolver in the course of our experiments.

\section{REFERENCES}

[Anderson et al. 1990] D. M. Anderson, H. T. Davis, J. C. C. Nitsche, and L. E. Scriven, "Periodic surfaces of prescribed mean curvature", Adv. Chem. Phys. $\mathbf{7 7}$ (1990), 337-396.

[Athanassenas 1987] M. Athanassenas, "A variational problem for constant mean curvature surfaces with free boundary", J. Reine Angew. Math. 377 (1987), 97-107.

[Brakke 1992] K. A. Brakke, "The surface evolver", Experiment. Math. 1:2 (1992), 141-165.

[Brakke 1994] K. Brakke, Surface Evolver Manual, Minneapolis: Geometry Center, University of Minnesota, 1994. See http://www.geom.umn.edu/ locate/evolver.

[Dubois Violette and Pansu 1990] E. Dubois Violette and B. Pansu (editors), International Workshop on
Geometry and Interfaces, Les Éditions de Physique, Orsay, 1990. Also J. Physique 51 (1990), no. 23 Colloq. C-7, suppl.

[Förster et al. 1994] S. Förster, A. K. Khandpur, J. Zhao, F. Bates, I. W. Hamley, A. J. Ryan, and W. Bras, "Complex phase behavior of polyisoprene-polystyrene diblock copolymers near the order-disorder transition", Macromolecules $\mathbf{2 7}$ (1994), 6922-6935.

[Große-Brauckmann 1993] K. Große-Brauckmann, "New surfaces of constant mean curvature", Math. Z. 214:4 (1993), 527-565.

[Grosse-Brauckmann 1996] K. Grosse-Brauckmann, "Stable constant mean curvature surfaces minimize area", Pacific J. Math. 175:2 (1996), 527-534.

[Große-Brauckmann 1997] K. Große-Brauckmann, "On gyroid interfaces", J. Colloid and Interface Sc. 187 (1997), 418-428.

[Große-Brauckmann and Kusner 1996] K. GroßeBrauckmann and R. Kusner, "Moduli spaces of embedded constant mean curvature surfaces with few ends and special symmetry", preprint, SFB 256, Universität Bonn, 1996.

[Große-Brauckmann and Polthier 1996] K. GroßeBrauckmann and K. Polthier, "Numerical examples of compact surfaces of constant mean curvature", pp. 23-46 in Elliptic and parabolic methods in geometry, edited by B. Chow et al., A K Peters, Wellesley, MA, 1996.

[Große-Brauckmann and Polthier 1997a] K. GroßeBrauckmann and K. Polthier, "Compact constant mean curvature surfaces with low genus", Experiment. Math. 6 (1997), 1-20.

[Große-Brauckmann and Polthier 1997b] K. GroßeBrauckmann and K. Polthier, "Constant mean curvature surfaces derived from Delaunay's and Wente's examples", pp. 119-134 in Visualization and mathematics, edited by H.-C. Hege and K. Polthier, Springer, Heidelberg, 1997.

[Große-Brauckmann and Wohlgemuth 1996] K. GroßeBrauckmann and M. Wohlgemuth, "The gyroid is embedded and has constant mean curvature companions", Calc. Var. Partial Differential Equations 4:6 (1996), 499-523. 
[Hajduk et al. 1994] D. A. Hajduk, P. E. Harper, S. M. Gruner, C. C. Honeker, G. Kim, E. L. Thomas, and L. J. Fetters, "The gyroid: A new equilibrium morphology in weakly segregated diblock copolymers", Macromolecules 27 (1994), 40634075 .

[Hsu et al. 1992] L. Hsu, R. Kusner, and J. Sullivan, "Minimizing the squared mean curvature integral for surfaces in space forms", Experiment. Math. 1:3 (1992), 190-207.

[Kapouleas 1990] N. Kapouleas, "Complete constant mean curvature surfaces in Euclidean three-space", Ann. of Math. (2) 131:2 (1990), 239-330.

[Karcher 1989] H. Karcher, "The triply periodic minimal surfaces of Alan Schoen and their constant mean curvature companions", Manuscripta Math. 64:3 (1989), 291-357.

[Korevaar and Kusner 1993] N. Korevaar and R. Kusner, "The global structure of constant mean curvature surfaces", Invent. Math. 114:2 (1993), $311-332$.

[Kusner 1991] R. Kusner, "Bubbles, conservation laws, and balanced diagrams", pp. 103-108 in Geometric analysis and computer graphics (Berkeley, CA, 1988), Math. Sci. Res. Inst. Publ. 17, Springer, New York, 1991.
[Lawson 1970] J. Lawson, H. Blaine, "Complete minimal surfaces in $S^{3}$ ", Ann. of Math. (2) 92 (1970), 335-374.

[Meeks 1990] W. H. Meeks, III, "The theory of triply periodic minimal surfaces", Indiana Univ. Math. J. 39:3 (1990), 877-936.

[Nitsche 1989] J. C. C. Nitsche, Lectures on minimal surfaces, Cambridge University Press, Cambridge, 1989. Translated from the German by Jerry M. Feinberg.

[Oberknapp and Polthier 1997] B. Oberknapp and K. Polthier, "An algorithm for discrete constant mean curvature surfaces", pp. 141-161 in Visualization and mathematics, edited by H.-C. Hege and K. Polthier, Springer, Heidelberg, 1997.

[Ross 1992] M. Ross, "Schwarz' $P$ and $D$ surfaces are stable", Differential Geom. Appl. 2:2 (1992), 179195.

[Schoen 1970] A. H. Schoen, "Infinite periodic minimal surfaces without selfintersections", Technical Note TN D-5541, NASA, 1970.

[Sullivan 1992] J. M. Sullivan, "VCS: software for computing Voronoi diagrams", 1992. See http:// www.geom.umn.edu/ sullivan/software/.

[Thomas et al. 1988] E. L. Thomas, D. M. Anderson, C. S. Henkee, and D. Hoffman, "Periodic areaminimizing surfaces in block copolymers", Nature 334 (1988), 598-601.

Karsten Große-Brauckmann, Universität Bonn, Mathematisches Institut, Beringstraße 1, 53115 Bonn, Germany (kgb@math.uni-bonn.de)

Received May 13, 1996; accepted in revised form October 25 